\title{
UBICACIÓN DEL INCISIVO SUPERIOR EN RELACIÓN A LA FRENTE Y PUNTO GLABELA EN PACIENTES DE LA CLÍNICA DE LA UNIVERSIDAD NORBERT WIENER
}

\author{
LOCATION UPPER INCISOR ABOUT THE FRONT \\ AND POINT GLABELLA IN PATIENTS OF THE CLINIC \\ OF NORBERT WIENER UNIVERSITY
}

\author{
LUIS ALBERTO HUÁRAC PÉREZ \\ Universidad Norbert Wiener \\ JUAN CARLOS FERNÁNDEZ VELASCO \\ Universidad Norbert Wiener
}

GINA LEÓN UNTIVEROS

Universidad Norbert Wiener

\section{RESUMEN}

La presente investigación se realizó con el fin de evaluar la ubicación del incisivo superior en relación a la frente y punto glabela en fotografías de perfil de los pacientes de la clínica de la Universidad Norbert Wiener. Se imprimió 30 fotografías extraorales de perfil en posición natural de la cabeza (PNC) en escala 1 x 1 para medir la ubicación del incisivo superior a través de una línea paralela a la vertical verdadera y que pase directamente por el punto glabela. Se encontró la siguiente ubicación de los incisivos en vista de perfil, en relación al punto glabela: en posición retruida $65 \%$, normal $15 \%$ y protruida $20 \%$. Se observa que los pacientes que acuden a la clínica y son sometidos a la evaluación de la fotografía de perfil extraoral presentan en mayor porcentaje retrusión del incisivo superior.

Palabras clave: incisivo, estética dental, maloclusiones.

\section{ABSTRACT}

This research was conducted in order to evaluate the location of the upper incisor in relation to the forehead and glabella point in profile pictures of clinic patients Norbert Wiener University. 30 profile extraoral photographs was printed in a Natural Head Position (NHP) in scale1 x 1 to measure the location of the upper incisor through a line parallel to the true vertical and passes directly by the glabela point. It was found that the location of the incisors in profile view in relation to the glabella point position retruded $65 \%$, normal $15 \%$ and $20 \%$ protruded. It is observed that the patients who come to the clinic and undergo assessment of extraoral photography profile are a higher percentage with upper incisor retrusión.

Key words: incisor, dental aesthetics, malocclusion. 


\section{INTRODUCCIÓN}

En la actualidad, el atractivo facial juega un rol importante en la interacción social. Esto influye en el éxito de atracción al sexo opuesto, las oportunidades de relacionarse, evaluaciones de personalidad, el rendimiento y las perspectivas de empleo. En la adaptación psicosocial, las personas con sonrisas hermosas son consideradas más atractivas, más inteligentes y más populares con respecto al sexo opuesto ${ }^{1}$.

La percepción de la belleza facial es multifactorial, con fundamento genético, ambiental y cultural. Es también valorada y clasificada de manera similar con respecto a la edad, género, origen étnico y estatus social. De tal manera que varía de persona en persona y está influenciada por las experiencias personales y el entorno social. Por tal motivo, la percepción fue definida como el proceso por el cual los patrones de estímulos ambientales están organizados e interpretados; esto puede ser influenciado por una variedad de factores físicos, fisiológicos y sociales ${ }^{2}$.

El atractivo facial y el atractivo de la sonrisa parecen estar firmemente relacionados entre sí. El hecho es que en la interacción social, la atención se dirige principalmente hacia la boca y los ojos del hablante. Como la boca es el centro de la comunicación en la cara, la sonrisa juega un papel importante en la expresión facial y la apariencia ${ }^{3}$.

La sonrisa es una de las más importantes expresiones faciales, y también es indispensable expresando términos de simpatía, aprobación y aprecio. Una sonrisa estética es el resultado de la interacción de los componentes de la sonrisa y requiere una comprensión de los principios que se manejan entre los dientes y los tejidos blandos ${ }^{4}$.
Una sonrisa estética requiere una integración perfecta de la composición facial y la composición dental. La composición facial incluye los tejidos duros y blandos de la cara. La composición dental se refiere más específicamente a los dientes y a su relación con los tejidos gingivales 5 .

Los incisivos maxilares, cuando son mostrados, deberían ser considerados como una parte de la cara, tanto desde una perspectiva frontal como lateral. El diagnóstico contemporáneo ortodóntico incluye la evaluación de la exposición de incisivos maxilares en una vista frontal. De perfil, sin embargo, los incisivos maxilares típicamente no son evaluados con respecto a su relación con la cara ${ }^{6}$.

La inclinación de la corona se refiere a una inclinación labiolingual o bucolingual del eje largo del mismo, no a la inclinación del eje largo de todo el diente. La importancia de la inclinación del incisivo es central para el diagnóstico y tratamiento ortodóntico. Además de jugar un importante papel funcional en la determinación de la estabilidad de la sobremordida, corregir su inclinación contribuye a la apariencia del atractivo facial. Así, una diversidad de inclinaciones del incisivo influye en una variedad de aspectos, incluyendo la estética, la autopercepción del paciente, la función, la estabilidad y la fonética ${ }^{7}$.

El propósito del presente estudio fue evaluar la posición anteroposterior de los incisivos centrales superiores en fotografías de perfil de la sonrisa en pacientes de la clínica de la Universidad Norbert Wiener.

\section{MATERIALES Y MÉTODOS}

El presente estudio es de tipo descriptivo observacional. La muestra fue escogida 
al azar y en treinta fotografias de perfil de pacientes que acuden a la especialidad de ortodoncia de la clínica de la universidad Norbert Wiener.

La fotografía de perfil en sonrisa fue tomada en posición natural de la cabeza por los estudiantes de la especialidad de ortodoncia de la Universidad Norbert Wiener y se imprimió a una escala de 1 x 1 para luego ubicar el punto glabela.

Con ese propósito, se utilizó una regla que se colocó en paralelo a la cadena y se trasladó hasta contactar con el primer punto que está a nivel de la zona supraorbitaria, para luego trazar una línea paralela a la vertical verdadera (Figura 1).

\section{FIGURA 1}

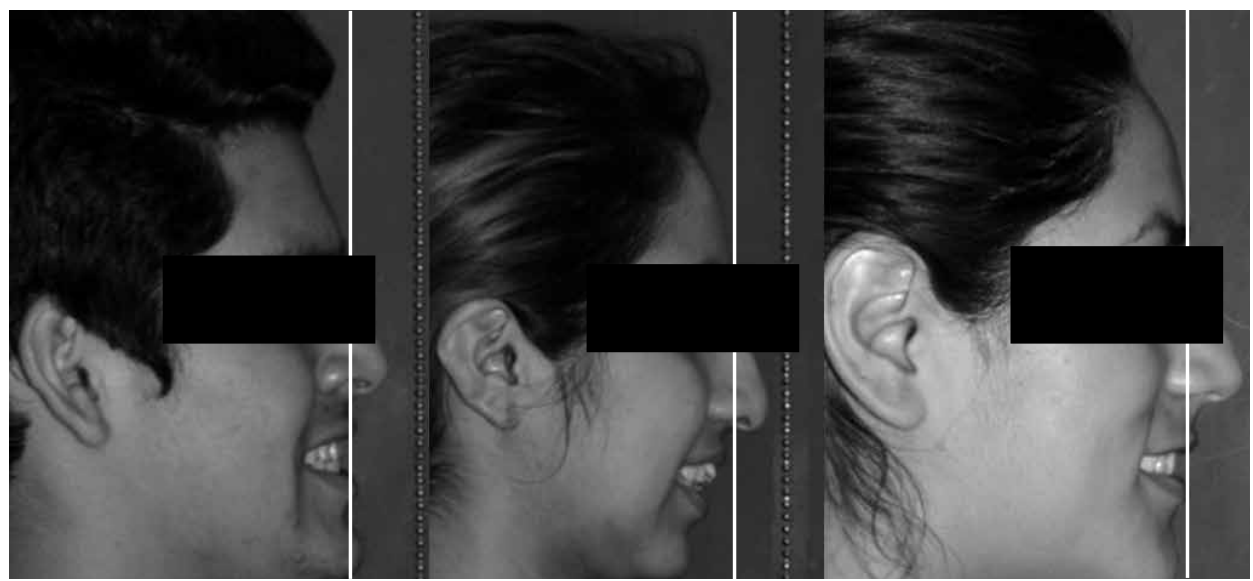

Finalmente, la ubicación anteroposterior de los incisivos centrales superiores fue establecida en función a su relación a la línea tangente al punto Gl. Cuando los incisivos estuvieron por detrás de esta línea fueron considerados como retruídos; cuando estuvieron a nivel de la línea tangente al punto Gl fueron considerados como normales; y cuando estuvieron por delante fueron considerados como protruidos.

\section{RESULTADOS}

En el presente estudio se observaron 30 fotografias extraorales de perfil en sonrisa, de las cuales $23 \%$ pertenecieron al sexo masculino y $77 \%$ al sexo femenino (Tabla 1 ).

En la evaluación de la posición del incisivo superior entre varones y mujeres se

TABLA 1

Ubicación de los incisivos superiores en relación al punto glabela

\begin{tabular}{|c|c|c|c|}
\hline & $\begin{array}{c}\text { RETRUIDO } \\
\mathrm{N}(\%)\end{array}$ & $\begin{array}{c}\text { NORMAL } \\
\mathrm{N}(\%)\end{array}$ & $\begin{array}{c}\text { PROTRUIDO } \\
\mathrm{N}(\%)\end{array}$ \\
\hline VARONES & $3(10 \%)$ & $2(6,7 \%)$ & $2(6,7 \%)$ \\
\hline MUJERES & $15(50 \%)$ & $3(10 \%)$ & $5(16,6 \%)$ \\
\hline
\end{tabular}

En la Tabla 1 se puede apreciar la comparación de la posición anteroposterior de los incisivos entre varones y mujeres. 
encontró en posición retruida $60 \%$, en posición normal $16,7 \%$, y protruida $23,3 \%$ (Gráfico 1).

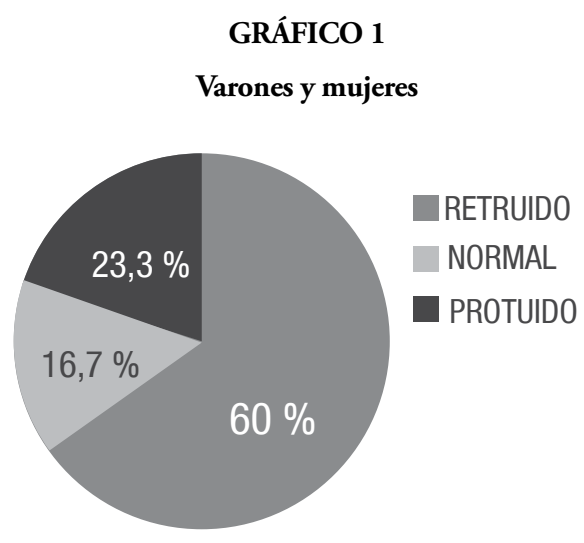

\section{DISCUSIÓN}

Hoy en día, el diagnóstico ortodóncico se encuentra orientado en base a la evaluación de los tejidos blandos como parámetro para la ubicación tridimensional de las estructuras dentarias y maxilares. Esta es la razón por la que durante una evaluación ortodóncica se debe incluir un detallado estudio de la estética facial, desde una perspectiva frontal y lateral ${ }^{8}$.

En la literatura ortodóncica se puede hallar las diferentes propuestas realizadas por autores que han tratado de describir métodos para evaluar el perfil facial de los pacientes. Uno de ellos fue el descrito por Andrews, quien en la década de los noventa presentó a los seis elementos de la armonía orofacial como un sistema de clasificación y metas terapéuticas?.

El análisis de Andrews utiliza puntos externos (en tejidos blandos) para la localización de dientes y maxilares, a diferencia de los métodos cefalométricos tradicionales que utilizan puntos internos (en tejidos óseos). Numerosos estudios reportaron la existencia de una gran variación para las posiciones de estos puntos internos. Además, no hay ninguna correlación segura entre las posiciones de los puntos internos y la posición anteroposterior óptima de los maxilares; si la hubiese, existiría solo un análisis cefalométrico utilizado por todos los ortodoncistas en lugar de los cientos que hay.

Parte importante de la propuesta de Andrews fue incorporar al uso de la frente como estructura de referencia para la ubicación anteroposterior de incisivos y maxilares. Andrews describió que entre la prominencia de la frente y la posición de incisivos y maxilares existe una correlación que permanece estable a lo largo de la vida. Posiciones óptimas anteroposteriores esqueléticas y dentarias están asociadas con la prominencia e inclinación de la frente, independientemente de la raza, edad, cultura o sexo. Cuanto más prominente sea la frente, más prominente serán los dientes y maxilares, en cuanto más inclinada sea la frente, más prominentes serán los dientes y maxilares.

Para comprobar esto, Andrews realizó un estudio en mujeres caucásicas que presentaban armonía facial y comprobó que el punto EV de los incisivos superiores no se hallaba por detrás del eje vestibular de la frente, ni más prominente que la glabela.

Por otra parte, Estrada ${ }^{10}$ propuso evaluar la posición anteroposterior de los incisivos superiores en relación a la frente en individuos peruanos de las comunidades de Hayuni, en el Cusco, y la comunidad de Taquile, en Puno. Los resultados del estudio muestran que en varones y mujeres la posición normal fue la más 
frecuente, seguida por la posición retrui$\mathrm{da}$, mientras que la posición protruida no fue hallada en ningún individuo.

Los resultados de los estudios de Andrews y Estrada coincidieron; sin embargo, el estudio realizado en fotografías de perfil de la clínica de la Universidad Norbert Wiener mostraron que la posición más frecuente es la retruida, seguida por la protruida, y luego, la posición normal.

Comparativamente, los resultados entre estudios anteriores y lo mostrado en el presente estudio muestran diferencia, por lo que se sugiere para estudios posteriores la estandarización de la muestra en la fotografía de perfil con posición natural de la cabeza.

\section{CONCLUSIÓN}

Existe una diferencia entre los estudios de Andrews y Estrada en comparación con los estudios realizados en la Clínica de la Universidad Norbert Wiener.

De la muestra se aprecia que los pacientes que acuden a la clínica de la Universidad Norbert Wiener registran generalmente la posición del incisivo retruido.

\section{REFERENCIAS BIBLIOGRÁFICAS}

1. Van der Gelda P, Oosterveldb P, Van Heckc G, Kuijpers-Jagtmand AM. Smile Attractiveness: Self-perception and Infl uence on Personality. Angle Orthod. 2007; 77(5):759-65.

2. Ghaleb N, Bouserhal J, Bassil-Nassif N. Aesthetic evaluation of profi le incisor inclination. Eur J Orthod. 2011; 33(3):228-35.
3. Abu Alhaija ES, Al-Shamsi NO, Al-Khateeb S. Perceptions of Jordanian laypersons and dental professionals to altered smile aesthetics. Eur J Orthod. 2011; 33(4):450-6.

4. Abu Arqoub SH, Al-Khateeb SN. Perception of facial profi le attractiveness of different antero-posterior and vertical proportions. Eur J Orthod. 2011; 33(1):103-11.

5. Cao L, Zhang K, Bai D, Jing Y, Guo Y. Effect of maxillary incisor labiolingual inclination and anteroposterior position on smiling profi le esthetics. Angle Orthod. 2011; 81(1):121-29.

6. McLeod C, Fields HW, Hechter F, Wiltshire W, Christensen J. Esthetics and smile characteristics evaluated by laypersons. Angle Orthod. 2011; 81(2):198-205.

7. Durgekar SG, Naik V. the ideal smile and its orthodontic implications. World J Orthod 2010; 11(3):211-20.

8. Bhuvaneswaran M. Principles of smile design. J Conserv Dent. 2010; 13(4):224-32.

9. Andrews WA. Anteroposterior relationship of the maxillary central incisor to the forehead in adult White females. Angle Orthod. 2008; 78 (4):662-69.

10. Estrada MA. Posición antero posterior de incisivos centrales superiores respecto al eje facial de la frente y Glabela en pobladores de las comunidades de Hayuni y Taquile, Cusco-Puno 2009 [Tesis]. Lima: Universidad Peruana Cayetano Heredia. 